EUROPEAN ORGANIZATION FOR NUCLEAR RESEARCH

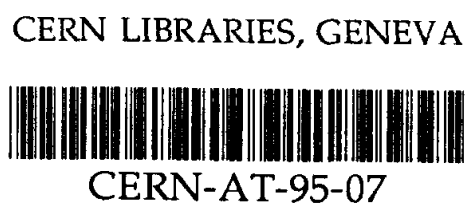

CERN / AT /95-07 (ET)

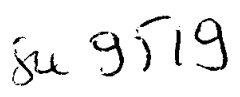

\title{
High efficiency photo cathodes for gaseous detectors of visible photons
}

\author{
by \\ V. Peskov*, \\ CERN, Geneva, Switzerland \\ and E. Silin \\ Inst. of Applied Mechanics, Russia \\ ( ${ }^{*}$ on leave of absence from Fermilab)
}

\begin{abstract}
Our latest results on the development of gaseous detectors of visible photons are presented. Quantum efficiencies of a few $\%$ at $400 \mathrm{~nm}$ were routinely achieved. These detectors can operate at gas gains up to $\sim 1000$. The main effort was focused on a study of the effect of different liquids and solid layers on the quantum efficiency of photo cathodes. Some of these layers make the photo cathodes more robust, while others increase the quantum efficiency. It was found, for example, that thin layers of solid and liquid $\mathrm{Xe}$ and $\mathrm{Kr}$ enhance the quantum efficiency by a factor $\sim 2$. These solid layers can also protect the photo cathodes against some impurities in the surrounding gas.

Probably after some further development gaseous detectors of visible photons will find applications in crystal calorimetry, noble liquid scintillation detectors, Cherenkov counters, air shower detectors and the readout of scintillating fibers for pre-shower and max-shower detectors.
\end{abstract}

Paper presented at the Vienna Wirechamber Conference, Vienna, February, 1995

Geneva, 12th April 1995 


\section{Introduction}

Proposals to use CsI photo cathodes in parallel-plate [1-3] and wire chambers [4] triggered a lot of interest in this type of photosensitive detector (see for example reports on this Vienna Wirechamber Conference). The main advantage of detectors with solid photo cathodes, compared to detectors filled with photosensitive vapours, is that they have very good time resolution $(<0.5 \mathrm{~ns})$ [5] as the photoelectrons are produced at a fixed distance from the anode. The parallel-plate design is less sensitive to direct ionization than traditional detectors filled with photosensitive vapours and this may have an advantage in some experiments. Solid photo cathodes allow one to build a new generation of gaseous detectors [6]. Rather big prototypes of RICH with CsI photo cathodes were recently built and successfully tested by different groups as can be seen in the Proceedings of this Conference.

CsI photo cathodes were successfully used also for the detection of the scintillation light from crystal scintillators [2] as well as from the gaseous, liquid and solid noble gases [4,7]. It was discovered that the quantum efficiency $(\mathrm{QE})$ of the CSI photo cathodes covered by a layer of liquid or solid $\mathrm{Xe}$ and $\mathrm{Kr}$ increases by a factor 2-4 compared to the original value in vacuum [7]. This may open new possibilities in the readout of noble liquid scintillating calorimeters and gamma detectors.

Unfortunately CsI is sensitive only for wavelength $<220 \mathrm{~nm}$ and this restricts the applications. It would be very important to increase the sensitivity of solid photo cathodes to longer wavelength. A few successful attempts have been done to combine photo cathodes sensitive to visible light with gaseous detectors (where the photo cathode is fabricated inside the detector) [8,9]. A QE of a few \% was achieved with a simple technique. A QE of $10-15 \%$ (which is not far from the photo multiplier sensitivity) was achieved in ref.[10,11] with a more sophisticated and cleaner gas system.

In this work we tried to reproduce the high $Q E$ reported in ref. $[10,11]$ and also to study the possibility to enhance the QE by covering the photo cathodes with additional liquid or solid layers. 


\section{Preparation of high efficiency photo cathodes}

Results from ref. $[10,11]$, as well as our own experience, show that the key to manufacture high QE photo cathodes is a vacuum better than $10^{-6}$ Torr and a very clean gas system. For these reasons in the present work maximum precautions were taken to ensure that our system is clean enough and made from appropriate materials, which have very low outgassing properties. Our set up contains a test chamber, a pumping system, a gas cleaning system, a light source with a set of filters and electronics. We had two types of test chambers: one made from glass and another from stainless steel. The highest QE (on 30 to $50 \%$ ) and most stable in time photo cathodes were obtained with the glass chambers, but most of the preliminary experiments were done with the stainless steel chamber since it has more flexible design allowing it to be opened to make changes inside and use it many times.

The schematic drawing of the glass test chamber is shown in Fig. 1a. It contains a glass vessel inside which was installed a parallel-plate chamber and a Cs generator. The anode of the counter was made from a stainless steel mesh, the cathode was a stainless steel disk. The distance between anode and cathode was typically $3 \mathrm{~mm}$.

The schematic drawing of the stainless steel chamber is shown in Fig. 1b. It has a flange with a sapphire window. Inside the test chamber the parallel-plate counter and a Cs generator were installed. The distance between anode and cathode was between 1-3 mm depending on the particular experiment. Only high vacuum materials were used : stainless steel, glass and ceramic.

In both chambers the stainless steel cathode disk was covered by a thin film $(1 \mu)$ of materials to be treated by Cs, for example: Sb or Au. In the case of GaAs and Si substrates they were mechanically attached to the cathode disk. Before the installation of these substrates the oxidation layer on their surface was chemically removed [12]. The cathode disk can be heated to $300^{\circ} \mathrm{C}$ or cooled down to Liquid nitrogen temperature separately from the whole system.

Before the photo cathode manufacturing the whole system was heated up to $250-300^{\circ} \mathrm{C}$ and pumped to a vacuum better than $10^{-6}$ Torr for a week or two. In order to additionally clean the surface to be coated ( $\mathrm{Au}$, $\mathrm{Sb}, \mathrm{GaAs}, \mathrm{Si}$ ) in some glass chambers after a week of pumping we would initiate a glow discharge in noble gas atmosphere at a few Torr (these 
chambers have an additional electrode not shown in Fig. 1). Pumping was resumed for another few days, after which the temperature was reduced to $150^{\circ} \mathrm{C}$ and the $\mathrm{Cs}$ generator was activated by passing a 5-8 A current through it. Cs vapours filled the entire volume of the test chamber. After a further few hours of pumping the Cs vapours were pumped out practically completely. The remaining $C s$ is that which has chemically reacted with the surfaces remained. The above is the procedure for producing our photo cathodes: Cs reacting with $\mathrm{Sb}$ or $\mathrm{Au}$ layers or with $\mathrm{Si}$ or GaAs wafer. We found that if everything was done according to this procedure then it is not even necessary to monitor the QE during the manufacturing (compare to [12]). However the cooling of the chamber and the whole system to room temperature should be done over a day or more. The reason is that devices in vacuum are well thermo-insulated and retain their temperature a very long time. Any temperature gradient allows the movement (by re-evapouration) of residual Cs from the chamber or pipes surfaces and this causes instability of the photo cathodes in time and may also spoil the QE.

We observed that if the system is well outgassed then the photo cathodes are stable even without a getter.

In some experiments, we tried to enhance the $\mathrm{QE}$ by introducing $\mathrm{O}_{2}$ [13]. In this case the QE was monitored in time and the oxidation was stopped when the QE reached the maximum.

After tests in vacuum we introduced different clean gases: Ar, Xe, $\mathrm{Kr}, \mathrm{CH}_{4}$, isobutane, DME or their mixtures.

As a light source a $\mathrm{Hg}$ lamp with a set of narrow band filters transparent only for the selected strong lines (185, 253, 297, 365, 405, 436 and $546 \mathrm{~nm}$ ) was used. The QE of our photo cathodes was measured at this wavelength with respect to calibrated photo tubes, obtained from Hamamatsu [12].

\section{Results in vacuum}

All photo cathodes tested have QE between few and 10\%. However the best results were achieved with $\mathrm{SbCs}$ and GaAs/Cs photo cathodes, manufactured inside the glass chamber and treated by a glow discharge. The typical QE achieved in vacuum is presented in Fig. 2. It should be noted that as in the case of commercial photo tubes the absolute value of the QE and the shape of the spectral response varied by $30-100 \%$ from 
photo cathode to photo cathode. In Fig. 2 only "typical" values are presented.

In the case of the $\mathrm{SbCs}$ photo cathode treated with $\mathrm{O}_{2}$, the $\mathrm{QE}$ was sometimes up to a factor 2 higher (up to $\sim 20 \%$ ), but the dispersion between different photo cathodes was very large and we need to learn how to get more reproducible results.

No ageing was observed at low flux, when the photo current was $<10^{-14} \mathrm{~A}$. We did these measurements up to total collected charge $\sim 10$ $\mathrm{mC} / \mathrm{cm}^{2}$. However at high flux (photo current $\sim \mathrm{pA}$ ) some variation of the $\mathrm{QE}$ with time was observed. This effect was more pronounced when the intensity of the source was varied with time, for example when it was blocked for some time. We attribute this to a so called "fatigue" effect [13].

Heating the photo cathodes to $80-100^{\circ} \mathrm{C}$ increased their QE. This effect can probably be used in practice as in the case of CSI photo cathodes [14].

\section{Test of different gases}

Our detector in vacuum can only work in collection mode. It is known however that some photo cathodes of visible light remain stable in some clean gases. There are two important advantages of gas filled detectors : they can work with some gain (at least up to 100 [8-11], and they are less sensitive to magnetic field than the one operating in vacuum[15].

We tested different gases: $\mathrm{Ar}, \mathrm{Xe}, \mathrm{Kr}, \mathrm{Ar}+\mathrm{CH}_{4}, \mathrm{Ar}+\mathrm{DME}$, Artisobutane. The highest gain, up to $3-4 * 10^{3}$, before a breakdown appears was achieved in DME and isobutane, however the QE was typically 30-50\% lower than the original value in vacuum (probably due to the impurities). In the case of $\mathrm{CH}_{4}$ the QE was as in vacuum (see Fig. 3), but the maximum gain was $\sim 1000$. The maximum gain was flux dependent: at high flux it was smaller. The maximum gain before discharge is quoted for rates $<10^{3}$ $\mathrm{Hz}$.

One can introduce another maximum gain: when a certain portion of feedback appears for example 10\% compared to the primary pulse. This gain with $10 \%$ feedback was naturally less- around 100 .

We studied also the ageing properties of our photo cathodes in different gases. In Fig. 4 is shown a relative photo current from the detector versus a collected charge. No ageing was observed in noble gases (the test was done up to collected charge $\sim 10 \mathrm{mC} / \mathrm{cm}^{2}$ ), with rather strong 
ageing in quenching gases, see Fig. 4. In mixtures of noble with quenching gases the rate of the $\mathrm{QE}$ degradation was proportional to the quencher concentration. One should note that the photo cathodes treated by the glow discharge or $\mathrm{O}_{2}$ had much worse ageing properties than one presented in Fig. 4.

\section{Coating of the photo cathodes by additional layers}

In this work one of our efforts was focused on a study of the effect of different liquids and solid layers on the quantum efficiency of photo cathodes, for example $\mathrm{CsI}$, Carbon, $\mathrm{Al}_{2} \mathrm{O}_{3}$ and others. As was shown before $[7,12,15,16]$ some of these layers make the photo cathodes more robust, some increase their quantum efficiency. The restriction on the volume of this article does not allow us to present all these data. We will describe briefly only the most surprising results obtained with noble liquid layers. We mentioned in the introduction that liquid and solid $\mathrm{Xe}$ and $\mathrm{Kr}$ layers on the top of the CsI photo cathodes increased their QE [7]. Coming from this experience we tried also to see if the enhancement can be achieved by covering our photo cathodes with layers of liquid and solid $\mathrm{Xe}$ and $\mathrm{Kr}$. For this purpose noble gases were introduced into the stainless steel chamber and the cathode was cooled so that condensation appears on its surface. Before introducing the noble gases into the chamber the gas was purified using an oxisorb and a cooled (alcohol with dry ice) ceolite trap. We passed the gas many times from one bottle to another [17]. For this purpose the bottle, which collected the gas, was cooled to Liquid Nitrogen temperature. After a few cycles of purification the chamber also was washed by the clean gas and then again pumped and the Cs generator operated. The chamber was then cooled and the noble gas slowly introduced into it. This allowed additionally to clean the gas. The cathode was then cooled. Depending on the cathode temperature a liquid or solid layer started to form on the surface. In the case of a solid layer we succeeded in obtaining rather thick layers. The layer could be more than the distance between anode and cathode. The photo current, normalized as the $\mathrm{QE}$, as a function of the voltage, in the case of Xe layer filling the space between anode and cathode is presented in Fig. 5. It is remarkable that the QE exceeds the vacuum level at some voltages. When we inverted the polarity the current was below the limit of our measurements, indicating that there is no false effect. The QE as a function of voltage for a layer thinner than the anode- 
cathodes distance is presented in Fig. 6 By comparing Figs. 5 and 6 one can see that for all thicknesses of solid and liquid $X e$ the $Q E$ exceeds the vacuum level at more or less the same voltages. For the very thin layer the QE was high even at low voltages. Probably the kinetic energy, obtained by the electrons after the photon adsorption was large enough for them to escape.

We also performed measurements when after the liquid layer formation on the top of the photo cathodes, some $\mathrm{CH}_{4}$ was additionally introduced into the chamber. This is a quenching mixture and allows us to work with some gas gain without feedback and spoiling the enhanced QEsee Fig. 6.

We found that a solid layer of Xe can serve also as a protective layer. When clean $\mathrm{N}_{2}$ was introduced into the chamber (for few minutes) and evacuated, practically no change happened to the QE. When we opened the valve for the dry air for a few seconds (modelling a leak), the QE dropped by 10 to $30 \%$. When we open the valve for normal air it dropped as shown in Fig. 7. The fact that the QE of the photo cathode covered by solid $X e$ is not affected by a short contact with $\mathrm{N}_{2}$ may open new possibilities: it allows us to do some manipulation with photo cathodes inside a clean glove box and simplify assembling more sophisticated detectors .

With $\mathrm{Kr}$ we found qualitatively similar results, but quantitatively the enhancement was much less - in the best case (solid $\mathrm{Kr}$ ) only 10 to $30 \%$ compared to the vacuum level.

The enhancement effect may open new possibilities in these photo cathode applications.

\section{Conclusion}

We demonstrated that gaseous detectors with high QE in the visible region of spectra can be built in the laboratory. The key in the quality of the photo cathode manufacturing is extreme cleanliness of the system Under this condition the photo cathodes are stable in time. No ageing was observed in vacuum up to a accumulating charge about $10 \mathrm{mC}$.

The main advantage of gas filled detectors compared to vacuum ones, for example vacuum tubes or devices of the type suggested by De Salvo [18] is insensitivity to magnetic field (few kG) often used in experiments[15]. No ageing was observed in noble gases. However rather 
strong ageing was measured in quenching gases. The ageing was roughly proportional to the concentration of the quencher. One of the best mixtures for the gaseous detector of visible photons is $\mathrm{Ar}+30 \% \mathrm{CH}_{4}$, which has relatively good ageing properties and allows to work with a gain up to about $10^{3}$.

We found that solid and liquid layer of $\mathrm{Xe}$ and $\mathrm{Kr}$ on the top of photo cathodes enhances the QE. The solid Xe layer can serve as a protective layer against the possible impurities in the gas.

We believe that after further developments the gaseous detectors of visible photons may find some applications for example in readout of crystal scintillators, noble liquid scintillating calorimeter, gamma cameras, Cherenkov counters, large surface air shower detectors and readout scintillating fibers for pre-shower and max-shower detectors.

\section{7. $\quad$ Acknowledgement}

We would like to thank Prof. G. Charpak, D. Hatzifotiadou and C. Williams for their friendly discussions and help.

\section{8. $\quad$ References}

[1] G. Charpak et al., Proceedings of Symp. on Particle Identification at High Luminosity Hadron Colliders, editors T. Gourlay and J. Morfin, Fermilab, Batavia, IL, 1989. p. 295.

[2] G. Charpak et al., "Study of $\mathrm{BaF}_{2}$ Calorimeters in Future Hadron Colliders" Perspective for New Detectors in Future Supercolliders, Erice, Italy, Oct. 17-24,1989, Preprint CERN-EP/90-41,1990.

[3] J. Seguinot et al., Nucl. Instr. and Meth. A297 (1990) 133.

[4] V. Dangendorf et al., Nucl. Instr. and Meth. A289 (1990) 322.

[5] G. Charpak et al Nucl. Instr. and Meth. A307 (1991) 63.

[6] I. Giomataris et al., Nucl. Instr. and Meth. A323 (1992) 434.

[7] E. Aprile et al., Nucl. Instr. and Meth. A338 (1994),328.

E. Aprile et al., Nucl. Instr. and Meth. A353 (1994) 55.

[8] V. Peskov, J. Appl. Spectr 48 (1988) 316.

[9] J.S. Edmens et al., Nucl. Instr. and Meth. A273 (1988) 145.

[10] D.R. Winn, IEEE Trans. Nucl. Sci. NS-36 (1989) 128.

[11] G. Charpak et al., Preprint CERN-EP/82-169 (1982).

[12] A. Borovik-Romanov et al., Nucl. Instr. and Meth. A348 (1994) 269. 
V. Peskov et al., Nucl. Instr. and Meth. A353 (1994) 184.

[13] A.H. Sommer "Photosensitive Materials" J. Wiley \& Sons Inc., NY 1968.

[14] D. Anderson et al., Fermilab Technical Memo TM-1753, Fermilab, 1991.

[15] S. Majewski et al., Preprint CERN-EP/83-89 (1983).

[16] A. Breskin et al., Preprint of the Weizmann Inst. WIS-94/47/Oct-PH, Rehovot, Israel, 1994.

[17] V. Peskov et al. "Stable operation of some Cs based photo cathodes inside liquid and solid Ar" to be published in the proceedings of the Nuclear Science Symposium IEEE-94, Orlando, Verginia, USA.

[18] R. De Salvo et al., Nucl. Instr. and Meth. A315 (1992) 375. 


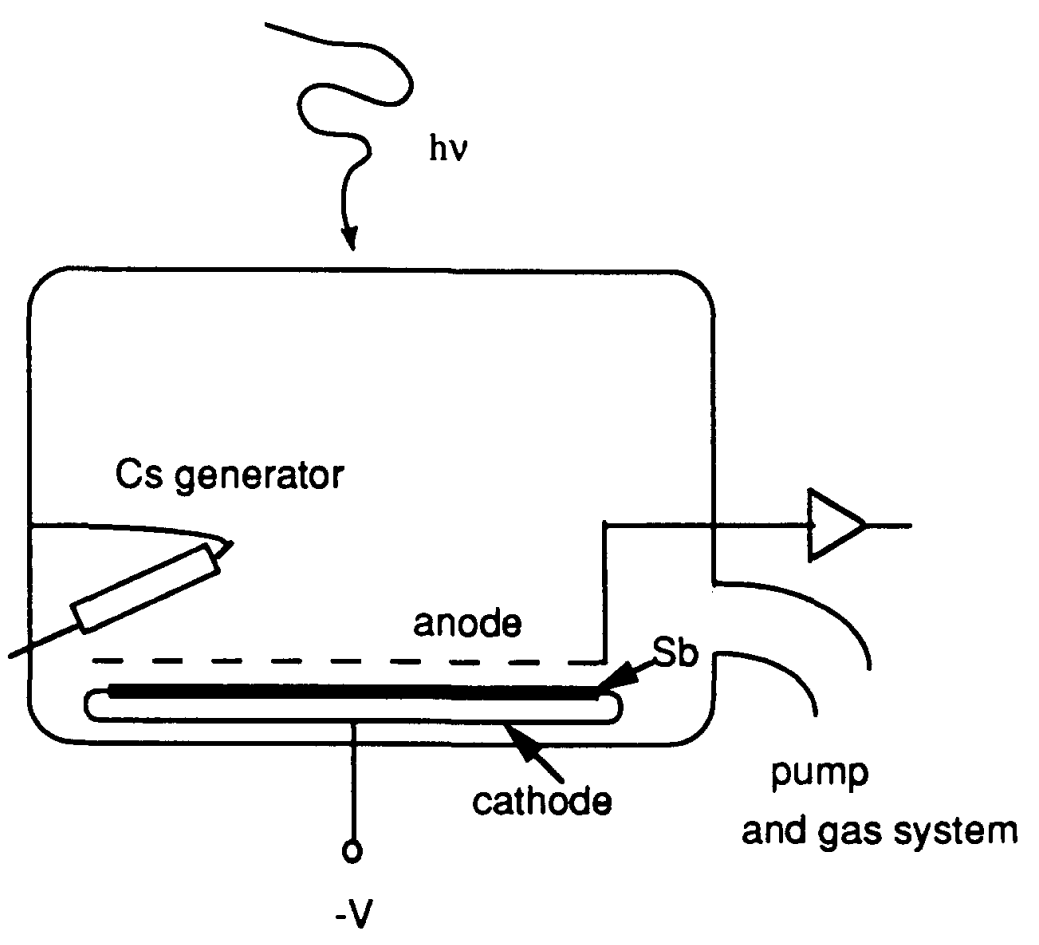

Fig. 1a Schematic drawing of the glass chamber 


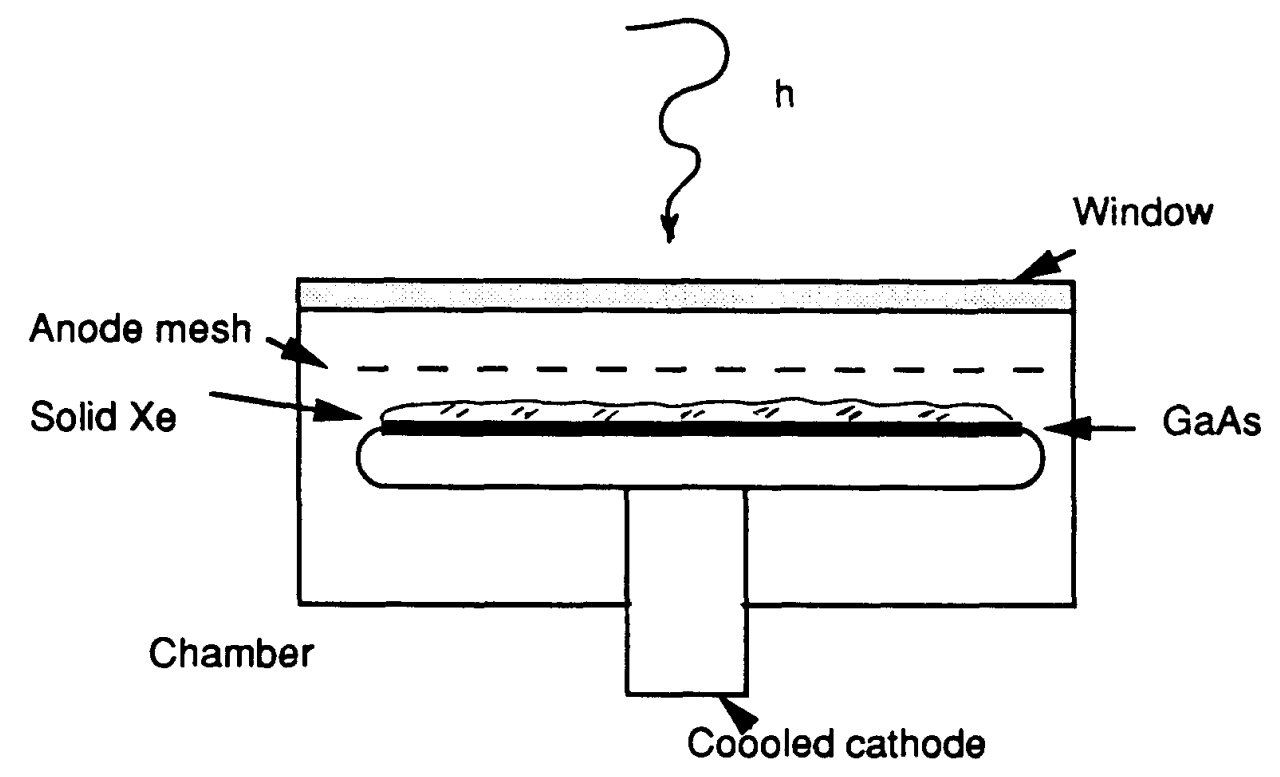

Fig. 1b Schematic drawing of the stainless steel test chamber 


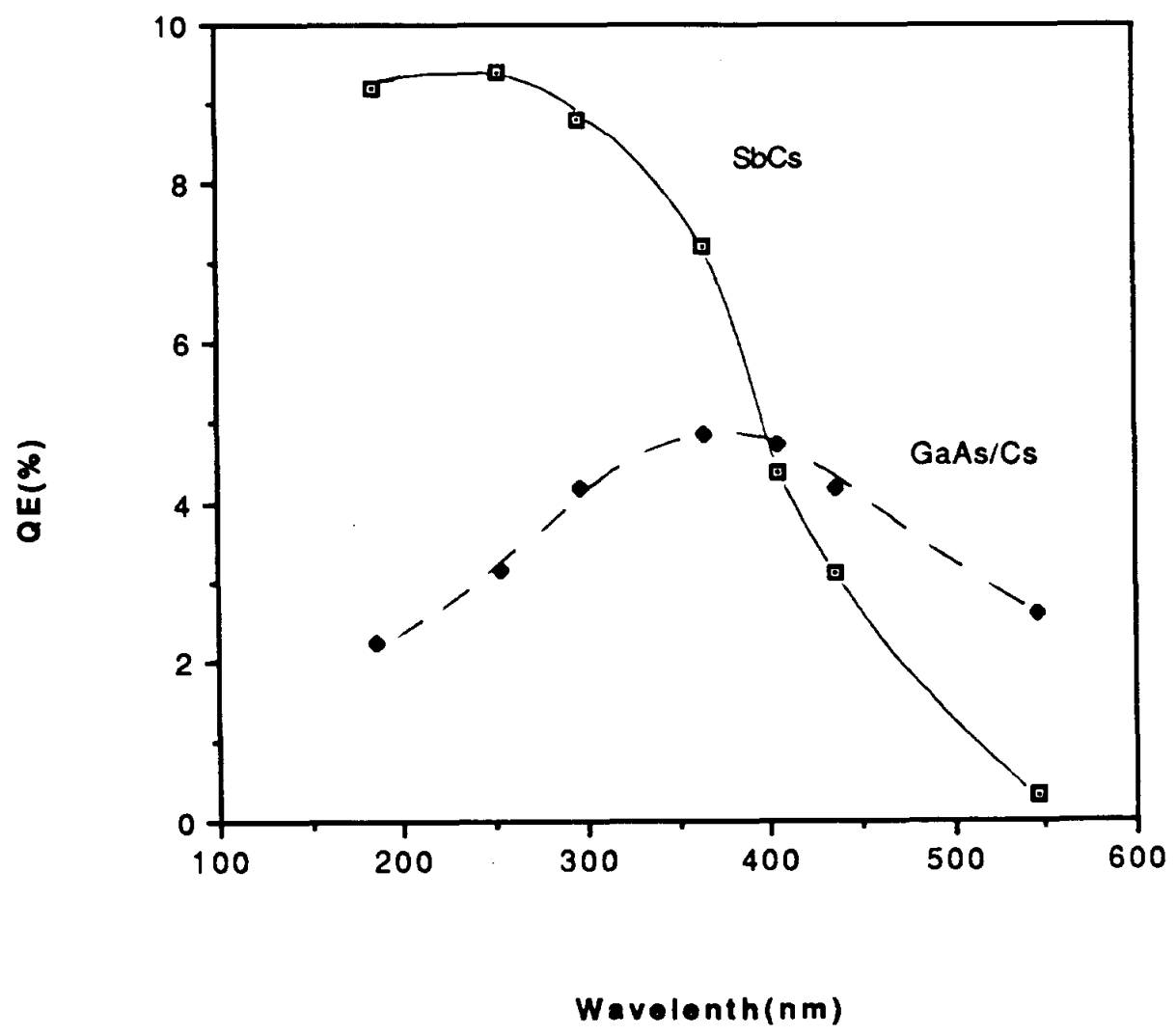

Fig. 2 Typical QE of SbCs and GaAs/Cs photo cathodes fabricated inside the glass chamber 


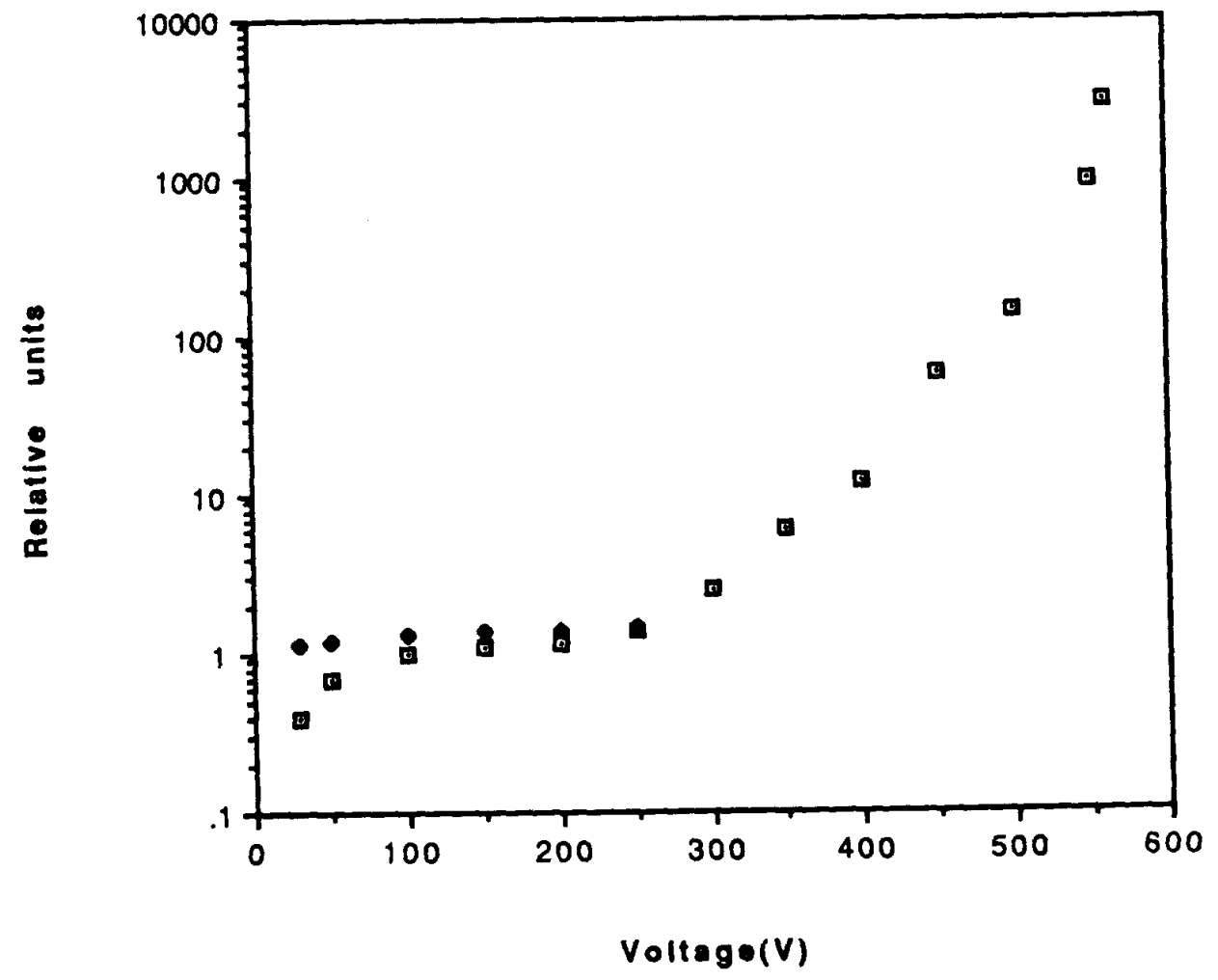

Fig. 3 Photo current in relative units versus voltage for the SbCs photo cathode fabricated inside the stainless steel chamber. 1-results in vacuum, 2- results in $\mathrm{CH}_{4}$ at 80 Torr. 


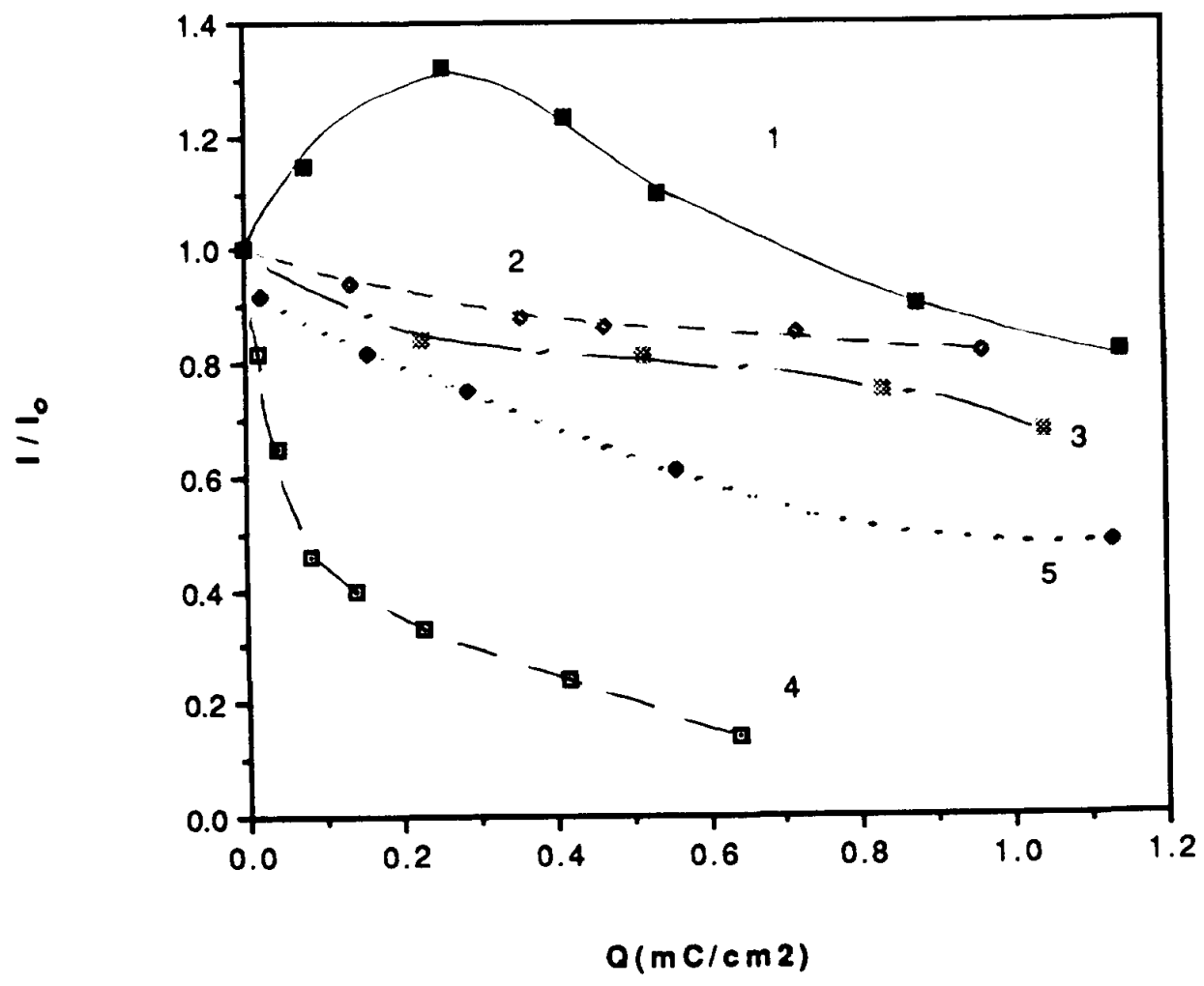

Fig. 4 Relative drop of the photo current(or $\mathrm{QE}$ ) versus collected charge for different conditions: $1-\mathrm{Ar}, 100$ Torr, $2-\mathrm{Ar}+10 \% \mathrm{DME}, 3-\mathrm{Ar}+30 \% \mathrm{CH}_{4}, 4-$ $\mathrm{CH}_{4}, 20$ Torr, 5-CH4, 20 Torr, the SbCs. photo cathode is covered by $20 \mathrm{~nm}$ CsI. All measurements were done at a gain 10 . 


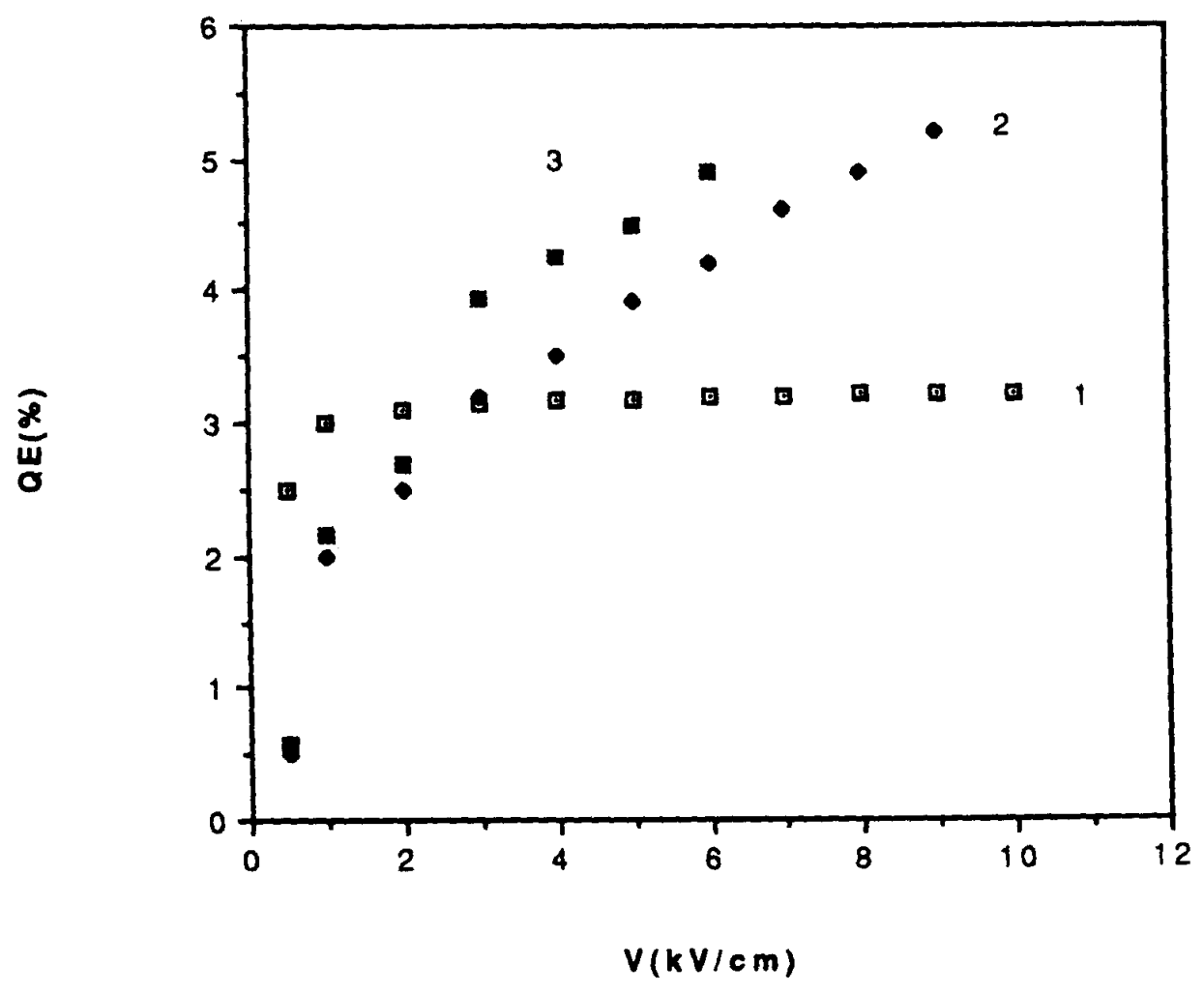

Fig. 5. $Q E$ vs. voltage for the $\mathrm{SbCs}$ photo cathode :1- in vacuum, 2-fully covered by LXe, 3- fully covered by SXe 


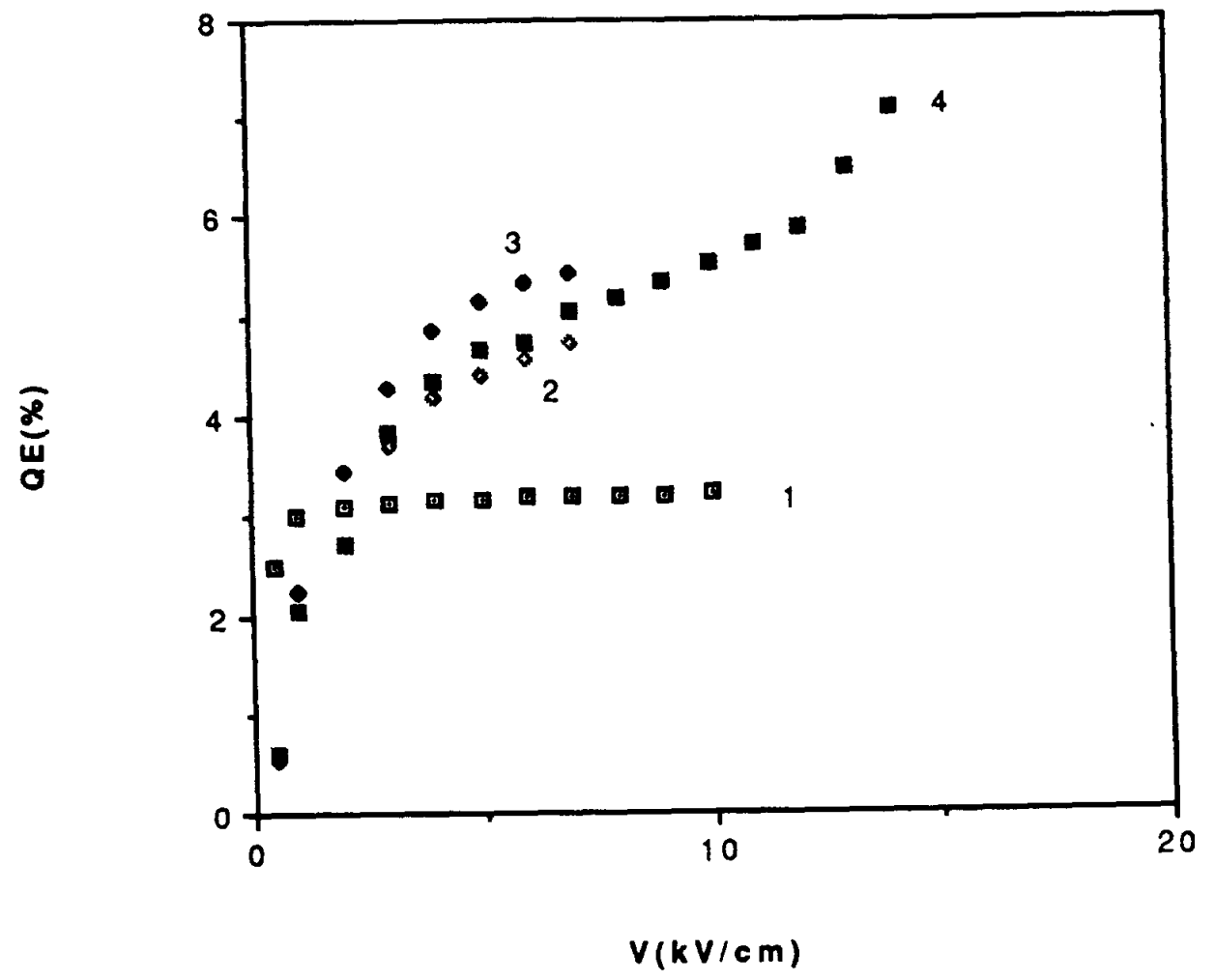

Fig. $6 \mathrm{QE}$ of the SbCs photo cathode :1-in vacuum, 2-covered by a thin layer of LXe, 3- covered by a thin layer of SXe. The pressure of gaseous Xe 0.7 Bar. The electric field correspondent the values inside the liquid or solid Xe.

4- Photo current when $\sim 0.05 \mathrm{Bar} \mathrm{CH}_{4}$ was added to the gaseous $\mathrm{Xe}$ 


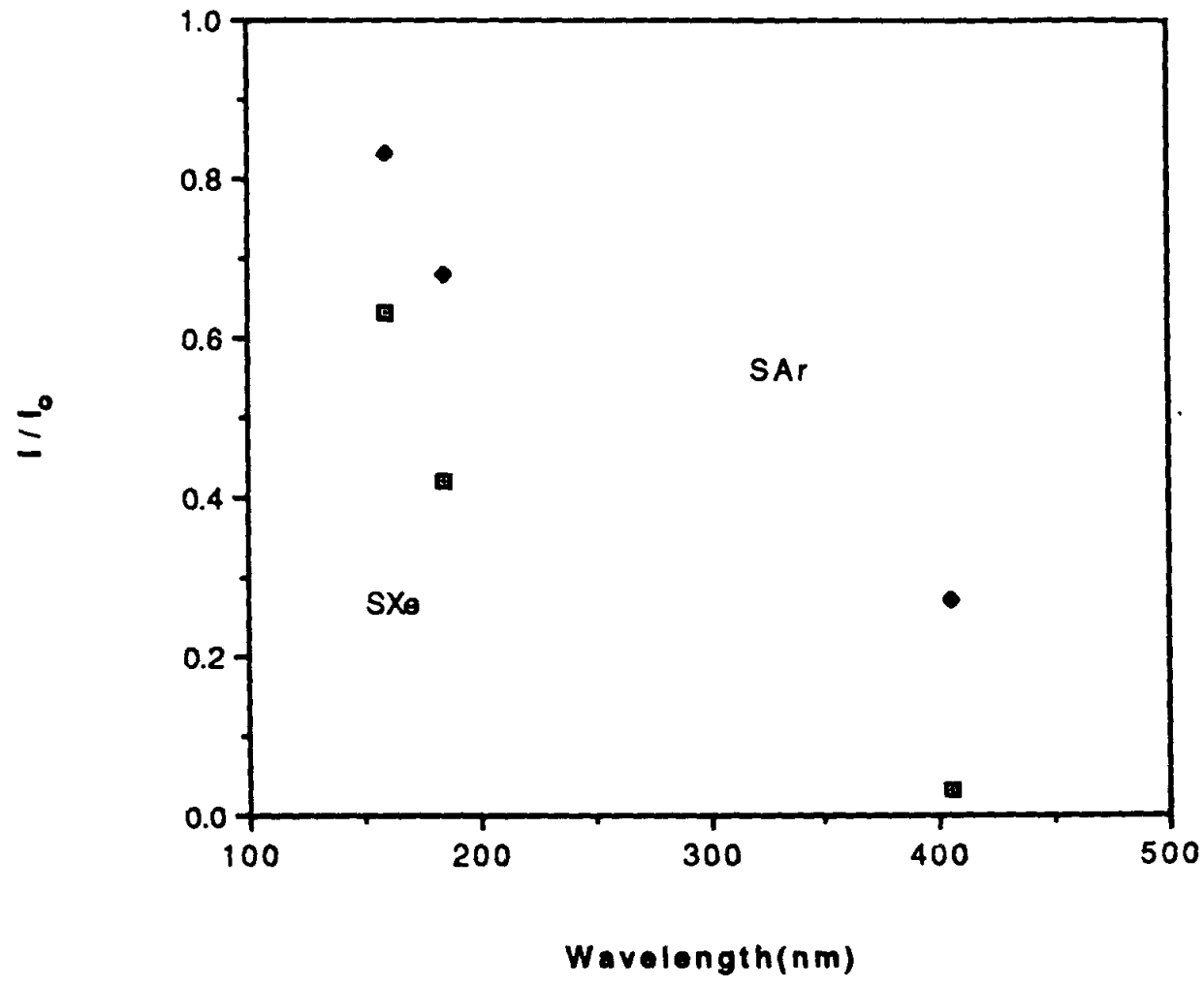

Fig. 7. Relative changes of the QE of the SbCs photo cathode, covered by a solid Xe and solid Ar after the valve of the chamber was opened to air for a few seconds 